\title{
The Child and Youth Profile: A Toolkit to Facilitate Cross-Disciplinary Educational Planning
}

Tara Way McLaughlin, Julia Budd and Sally Clendon

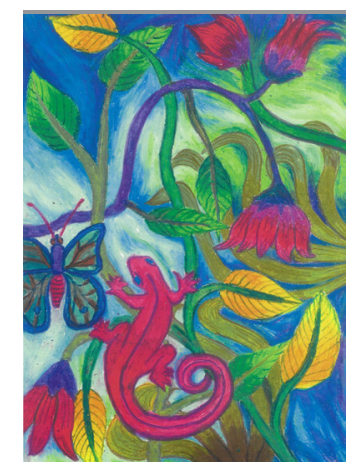

\begin{abstract}
Collaboration is an essential component of optimal educational planning. In order to collaborate effectively, it is important that information from all of those involved with the learner is used to develop a multi-dimensional understanding of the child/youth. Yet the identification, sharing and collating of this key information can be a difficult process due to lack of time, a focus on specialist jargon, and lack of tools or expertise. In this article, we introduce a toolkit, called the New Zealand Child and Youth Profile, to assist educational teams to discuss key aspects of children's functional experiences and abilities to inform educational planning. The toolkit is inspired by the International Classification of Functioning, Disability, and Health for Children and Youth (ICFCY) but contextualised for New Zealand. The toolkit has recently been piloted and revised based on user feedback and is available for teams interested in using it to facilitate cross-disciplinary educational planning.
\end{abstract}

\section{Research paper}

\section{Keywords:}

childhood disability, classification, collaboration, functional ability, ICF-CY

\section{INTRODUCTION}

It is well-documented that cross-disciplinary approaches to educational planning significantly improve learner outcomes (Demchack, Alden, Bergin, Ting \& Lacey, 1995). In line with this, the New Zealand Ministry of Education developed Collaboration for Success; guidelines which place collaboration between all of those involved with a student's learning at the heart of the educational planning process (Ministry of Education, 2011). Collaboration, however, is never easy, particularly when team members hold diverse perspectives (Conklin, 2005; Hinrichs, 2008). These multiple perspectives, however, are essential for optimal educational planning (Annan \& Mentis, 2013). Tools are needed to help teams to draw on and share their understanding with one another (Budd, 2016). One such tool is the New Zealand Child and Youth Profile; a toolkit which seeks to gather the multiple perspectives from those involved with the child/ youth and use them to facilitate collaboration for educational planning. This profile is discussed in this article.

In order to provide a context for the New Zealand Child and Youth Profile, this article begins with an introduction to different information-gathering and classification systems and their alignment with particular approaches to disability. This is followed by an introduction to the International Classification of Functioning, Disability, and Health for Children and Youth (ICF-CY) and an explanation of how it was used to guide the development of the New Zealand Child and Youth Profile. We then describe the toolkit and the pilot study to consider its usefulness and social validity. We end with a discussion on the recommended ways to use the toolkit, noting the opportunities and challenges for teachers, teams, and families interested in using it.

\section{SYSTEMS AND APPROACHES TO DISABLITY}

Information-gathering systems for children and youth with disabilities provide a way to organise complex information and promote efficient communication about children's abilities and limitations. Welldeveloped systems in education should lead to access to needed services and better-understanding of children and youth within the context of their environments. This, in turn, should inform educational planning that results in positive outcomes for development, learning, and transition to higher education or the workforce. To provide special educational services, systems that classify, categorise, and, often, label children with disabilities are often used to access services or funds for programmes (Simeonsson et al., 2003). Within New Zealand, one of these systems is the Ongoing Resourcing Scheme (ORS) in which children receive ORS classifications in order to be eligible for services. Despite their necessity, these classification systems provide insufficient information to guide educational planning and may perpetuate assumptions about 
children's abilities or lowered expectations for education attainment (Burke \& Ruedel, 2008). Thus, educational teams need additional information-gathering systems to support educational planning.

In the past 50 years, different systems and models have been in and out of favour amongst professionals and families. For example, past approaches have been based on a medical or deficit model in which children's characteristics, abilities and limitations are examined or evaluated as symptoms of a condition or deficit in development that results in "diagnosis" or assignment of a disability category, suggesting a biological or psychological etiology of disability (Burke \& Ruedel, 2008). The medical or deficit model has been criticised as it places the origin of disability within the child, without reference to the contributions of social or environmental factors, and focuses on the child's deficits and inabilities rather than the child's strengths and abilities.

Following dissatisfaction with a medical or deficit model, disability advocates and researchers promoted the need for a social or ecological model of disability that emphasises the social or environmental barriers that result in a child experiencing a disability or limitation (Simeonsson, 2009). These models focus on social attitudes or environmental barriers that inhibit the full participation of children within school and the community. The utility of social or ecological models, however, can be affected by a one-dimensional view such that the contributions of biological or psychological factors associated with disability might not be considered (Terzi, 2008). Increasingly, favouring one model to the exclusion of other models has been viewed as inadequate to account for the complex interactions among factors that affect children's health, development and functioning within and across contexts (cf. AAID, 2010; WHO, 2007).

In response to concerns with disability classification systems that are driven by previous (one-dimensional) models, the International Classification of Functioning, Disability, and Health for Children and Youth (ICF-CY) framework described by the World Health Organization (WHO, 2007) incorporates useful aspects of the medical, social and ecological models of disability into a biopsychosocial model (Peterson, 2005). This model emphasises the interactions among biological, psychological and social factors within the context of the environment (Cameron, 2008). The ICF-CY is an international unifying framework and classification taxonomy that is used to conceptualise childhood/ youth disability from a multi-dimensional perspective, emphasising the functional impact of disability, strengths and abilities, and participation in everyday settings.

\section{THE ICF-CY FRAMEWORK}

The focus on function distinguishes the ICF-CY from other frameworks and classification systems of childhood/youth disability. In addition, a lifespan approach acknowledges that as children's environments and developmental abilities change from birth through childhood, adolescence, and into adulthood, the nature and consequence of the person-social-environment interactions will change over time as well (Simeonsson et al., 2003). The ICF$\mathrm{CY}$ framework can complement existing systems of health conditions and disability to help promote a more holistic view of children's health, development and functioning.

Within the ICF-CY framework, a medical diagnosis or health condition interfaces with key components related to functioning and disability. These components can be viewed from a positive perspective under the term "functioning" and include the integrity of body functions (i.e. physiological and psychological), body structure (i.e. anatomical parts), activities (i.e. tasks a child completes), and participation (i.e. the integration of activities in life). Alternatively, these same components can be viewed from a negative perspective under the term "disability" and include impairment of body function, impairment of body structure, activity limitation, and participation restriction (Cramm, Aiken \& Stewart, 2012; WHO, 2007). The framework highlights the influence of contextual factors, including both environmental factors (e.g. physical, social and attitudinal environment) and personal factors (e.g. age, gender, ethnicity) on an individual's overall wellbeing and adaptation with regard to human functioning and restrictions on functioning (WHO, 2007).

The ICF-CY provides a structure to conceptualise and examine the functional impact of disability across the body/mind, the person, and society. From this multi-dimensional view, functioning can be affected by any interactional combination of impairment related to the body/mind, limitation related to the person's activities, or disadvantage related to participation in society (Cramm, Aiken \& Stewart, 2012). The ICF-CY outlines core areas of functioning and provides guidance on acknowledging and accommodating them into thinking, practice, and assessment (Rosenbaum \& Stewart, 2004). For practical application, the ICF-CY introduces a pre-specified taxonomy and numeric coding system that can be used to document the functional impact of disability across the identified areas of the framework. 
The ICF-CY has both conceptual (framework) and practical (classification taxonomy) appeal for describing and documenting childhood disability. The ICF-CY, however, is not an assessment tool, but rather a framework and classification taxonomy to guide thinking and the development or selection of assessment measures and the integration of functional information to enhance cross-disciplinary collaboration and educational planning (BjorckAkesson et al., 2010; Lollar \& Simeonsson, 2005; Simeonsson, 2009).

\section{THE NEW ZEALAND CHILD AND YOUTH PROFILE}

In response to increased awareness of the ICF-CY and the importance of a functional approach to assessment and educational planning, our team developed a toolkit referred to as the New Zealand Child and Youth Profile. We developed the toolkit for use in a range of New Zealand based settings during 2015. The toolkit was designed to collate information about a child's strengths and limitations; sensory function; participation in home, school and community settings; and interests and preferences. The information gained from the different parts of the toolkit supports a multi-dimensional perspective of children's educational needs and strengths that can facilitate cross-disciplinary collaboration and educational planning for children and youth with disabilities. As part of the development process, we engaged in expert review and feedback. In 2016 we piloted the use of the toolkit with a range of educational teams. The pilot study was designed to gather stakeholder feedback on the usefulness and social validity of the toolkit and inform revisions. In the remaining sections we outline the pilot study, discuss the key findings and next steps, and make recommendations for the future use of the New Zealand Child and Youth Profile.

\section{PILOT STUDY}

The Child and Youth Profile was designed as a toolkit to support teachers, families and specialists to reflect on and organise their understanding of a child's needs, abilities and participation in everyday settings, and consider different factors that might affect a child's functioning and outcomes. We conducted a pilot study to gather stakeholder feedback. This was guided by the following research questions:

1. How do different stakeholders (teachers, education professionals, and parents) use the Child and Youth Profile?

2. To what extent is the Child and Youth Profile useful in educational planning?
3. To what extent is the Child and Youth Profile socially valid?

Social validity referred to the extent to which the toolkit was useful, appropriate, culturally-relevant, feasible to use, and effective for supporting the team with educational planning.

\section{Participant recruitment}

Recruitment was based on professional contacts of the authors targeting teachers and teams that work with children with disabilities in early childhood and school-age settings. Professional contacts were invited to share the toolkit with teams of teachers, other education professionals and families (all referred to as stakeholders) for educational planning. After sharing the toolkit, we then invited teams to participate in our pilot study. Team member participation was voluntary and all participating members provided their informed consent.

\section{Participants}

The toolkit was shared with professionals at four educational settings. Three of these settings agreed to participate in the pilot study. Setting A was a special school in an urban area serving children with disabilities aged 5-21 years. In this setting, all teachers and specialists were introduced to the toolkit. From this, one administrator, one teacher and four specialists (two speech-language therapists, two occupational therapists) engaged in using the toolkit with two children. Setting B was an early intervention centre in an urban area serving children with disabilities aged birth through five years. In this setting, all teachers and specialists were introduced to the toolkit, and one teacher and one specialist (speech-language therapist) used it individually with families. Setting C was an educational resource centre where itinerant teachers provide specialist support to children from birth through 21 years across a range of service types. In this setting, four specialist teachers were introduced to the toolkit and used it with four preschool children.

\section{The Toolkit}

The pilot version of the toolkit was comprised of several forms and scales. The toolkit included the following sections.

Background Information. This section was designed to provide information about a child's cultural identity, educational placement, Ongoing Resource Funding (ORS), the professionals who work with the child and family, family members, and family support services.

Sensory Modality Profile (SMP). The SMP was 
designed to gather information about a child's current level of ability to perceive sensory information and use it to explore their world, discriminate between objects and use it for specific tasks.

Communication Profile (CP). The CP was designed to gather key information about the child/youth's communication and record how the child/youth expresses different important communicative functions.

Participation and Access Profile (PAP). The PAP was designed to ascertain a child's interest, availability, access and accommodation to a range of educational, recreation and sport, family, cultural community and religious activities, and to consider if participation is acceptable to the child, family or others involved in the activity.

Functional Ability Profile (FAP). The FAP was designed to gather information about a child's current level of ability across 11 functional ability areas. Functional abilities are viewed as the integrated skills that children can do to perform the activities of life (McLaughlin, Snyder \& Algina, 2015; Simeonsson, Bailey, Smith \& Buysse, 1995). The FAP helps to show areas of strength and weakness for the child/youth.

Adaptations and Specialised Equipment Profile (ASEP). The ASEP was designed to identify the types of adaptations, augmentative and alternative communication systems, assistive technology, or adaptive devices the child currently uses and/or which might be desirable for future use.

Interests and Preference Profile (IPP). The IPP was designed to identify a child's areas of interest and preferences for items and activities so that they can be used as starting points to increase engagement and participation, and develop meaningful supports for children. This includes an awareness of family preferences for the child.

Priority Planning Pages (PPP). The PPP were designed to integrate information from the toolkit to inform planning and interventions. Key information about the child can be documented in one page, and a second page can be used to list priority goals and interventions for home, school and community.

\section{Training and procedures}

A semi-structured training was provided to introduce the toolkit. This included background information and an overview of the ICF-CY to set the context for the toolkit. We also walked participants through the toolkit noting the purpose and features of each individual section. Finally, we outlined the purpose and process for the pilot study - noting that teams should use the toolkit as they saw fit, and that researchers would return in a few months time to gather information about their experiences. It is important to note that the researchers intentionally did not give information about how teams might or should use the toolkit. Guided by the first research question, we were keen to examine how teams might use the toolkit without providing specific procedures or direct guidance.

\section{Data collection}

During the initial semi-structured trainings, the researchers took notes about participants' questions and comments to help inform future trainings and focus group/interview questions. Following the training and after two to three months of use, the participants engaged in focus group interviews at each setting. The focus group interviews were semistructured and led by at least one researcher. They were designed to elicit stakeholders' perspectives about how they used the toolkit and the extent to which it was useful and socially valid. The focus group interviews lasted between 45 and 75 minutes. The researchers did not have access to the children's data that was collected using the toolkit.

\section{Data analysis}

The focus groups interviews were transcribed for analysis. Each researcher took the lead on the analysis for one setting. The transcripts and researcher notes were analysed for specific recommendations for revisions and key themes related to the participants' feedback and experience. The researchers met to discuss recommendations and key themes from each setting as well as identify shared experiences or differences based on the diverse ways in which the toolkit was used at each setting.

\section{FINDINGS}

The findings are organised into four sections how the toolkit was used, perspectives on social validity, specific recommendations for revisions, and challenges and opportunities. As noted earlier, social validity was intended to be inclusive of the extent to which the toolkit was useful, appropriate, culturally-relevant, feasible to use, and effective for supporting the team with educational planning.

\section{How the Toolkit was Used}

The toolkit was used in different ways at each of the settings. Notably, several participants reported using it differently than they had originally envisaged or expected. At Setting A, the team used the toolkit for two children undergoing transition. For this team, one person took responsibility for compiling all of 
the notes and the information about the child and transferring the information onto the toolkit forms and profiles. At Setting B, the two staff members used it individually with families. One worked with a mother and father to complete the forms and profiles together, while the other asked the mother to complete the toolkit and then reviewed it with the mother afterwards. At Setting C, the four teachers used the toolkit individually for their own planning purposes. Across sites, the teams and teachers completed or attempted to complete most of the forms and profiles. All of the teams commented on the utility of the summary form, but interestingly, the form for educational planning was not used by any of the teams.

\section{Perspectives on Social Validity.}

Two of the settings reported that the toolkit was useful for collating and organising a range of information, including information they had not previously considered or documented. One of these settings also found that it was useful for collating data from a range of assessments from different sources that could then be used to plan programmes. The remaining setting reported that they already had other tools and systems to document most of the information and were concerned that this was an unnecessary double-up. Team members from the two settings that felt it was useful also reported that it would be helpful for interdisciplinary sharing or collaborating with other team members, but none of the sites actually used it in this way.

Related to the specific parts of the toolkit, all teams reported that the key areas focused on across the forms and profiles were appropriate and relevant for children and youth with disabilities and no areas were identified as missing. Within specific forms and profiles, however, the teams noted that not all aspects were age-appropriate or relevant to the age group they focused on. For example, the early childhood participants noted that indicators on the participation and access scale for education settings did not seem relevant for early childhood. Related to cultural relevance, the participants noted that the cultural identity categories given in the background sheet were not sufficiently comprehensive and there was no space to record the child/youth's home language.

The participants reported that the toolkit forms were generally feasible to use; however, simple revisions such as the placement of directions and definitions at the beginning of the forms would improve the user's ability to complete them easily. The participants also reported wanting to adapt the forms for setting-specific information. One setting noted that it might be challenging to use the toolkit with the multiple forms and profiles at a team meeting with families as they try to avoid focusing on the "paper work" and allow for more natural conversation.

\section{Specific Recommendations for Revisions}

Specific recommendations for revisions on the toolkit ranged from basic typos to restructuring the order of the forms and profiles presented. There was a strong preference for more space for notes and comments related to the different sections, and more opportunities to personalise the form to the individual settings. Given the range of information collated, several participants noted the need for more clarity of terms or definitions. In addition, a key discussion area related to the use of ratings for different aspects of the toolkit. For example, for the Functional Ability Profile, some participants felt that it was not helpful to compare a child's ability to same-aged peers, while others found that it was hard to rate a child's ability in this way. Notably, the participants who used the form directly with the families found this rating to be unhelpful. Suggestions included rating the key areas but in terms of specified criteria or strengths and limitations within the child rather than in a comparative way.

\section{Challenges and Opportunities}

One of the most notable findings from our discussions with the participants was that they experienced both challenges and opportunities from using the toolkit. Common challenges included confusion about whether the toolkit was an assessment tool, the amount of time needed for completion, compatibility with setting-specific requirements or procedures, and knowing how to use it with families. For the settings that did not involve families in the process, they were unclear how completing the structured forms would work for families - preferring to use the toolkit as a reference point to plan and reflect on their conversations with families. For the setting that did use the form with families, they found that families were overwhelmed by the terms or unclear about the concepts. In addition to the challenges listed above, several participants described challenges with some of the structured ratings related to different areas of functioning. This was sometimes related to the notion of making a general rating and sometimes related to clarity about scale anchors or from whose perspective the rating should be made.

Despite the challenges, the participants also highlighted the opportunities and potential of the toolkit. Most notably, all settings noted that the 
toolkit made them think about and reflect on areas of child functioning that they had not previously considered. They described the toolkit as useful for broadening their thinking, becoming aware of other disciplines, or realising an important area that they had not thought about before. The participants who used the toolkit with others reported that they learned something they did not know about the child in a different context. They also reported the importance of documenting aspects of the child's abilities and needs that they might have known but had not formally documented or shared with others.

\section{DISCUSSION}

As researchers, we expected the settings to use the toolkit in diverse ways as well as experience challenges, particularly in the absence of more specific training or guidance about its use. We also recognised that each setting already had existing systems for collaboration and educational planning, and we intentionally did not provide guidance on how the toolkit might support or enhance these. Nonetheless, we wanted to explore how educational professionals might use the toolkit without this guidance, and understand whether the forms and profiles in the paper document were sufficient. We believe the pilot has indicated a resounding 'no'.

Although participants recognised that the toolkit would be useful to help facilitate cross-disciplinary collaboration, in line with the Ministry of Education's Collaboration for Success (Ministry of Education, 2011), none of the participants actually used the toolkit in this way. This finding is consistent with research related to collaboration in general as well as to the ICF-CY, which highlights the challenges of cross-disciplinary communication, information sharing, and meaningful involvement of families particularly where multiple perspectives are involved (Conklin, 2005; Hinrichs, 2008). Nonetheless, there is a need to bring together diverse sources of information and perspectives about a child's functioning in and across contexts to ensure that educational planning encompasses the needs of the whole child/youth and maximises learning potential. As researchers, we are interested in further exploring the reasons why the toolkit was not used in a collaborative way; was it due to the design and structure of the toolkit or because teams lacked time or resources, or needed more specific guidance and training to engage in cross-disciplinary teaming using a tool such as the Child and Youth Profile to draw out multiple perspectives?

Another area of challenge, and possible misunderstanding around the toolkit, was its intended use as a tool for collating information rather than being an assessment tool. Some of the participants, for example, stated that the toolkit did not provide enough space or opportunity for documenting information related to their own specialist area. More training is needed to help the teams to use the profile to collate summary information that might be informative to all team members rather than providing complete assessment information in particular areas. The framework in Figure 1 begins to outline how this might look and will be discussed further in the following section.

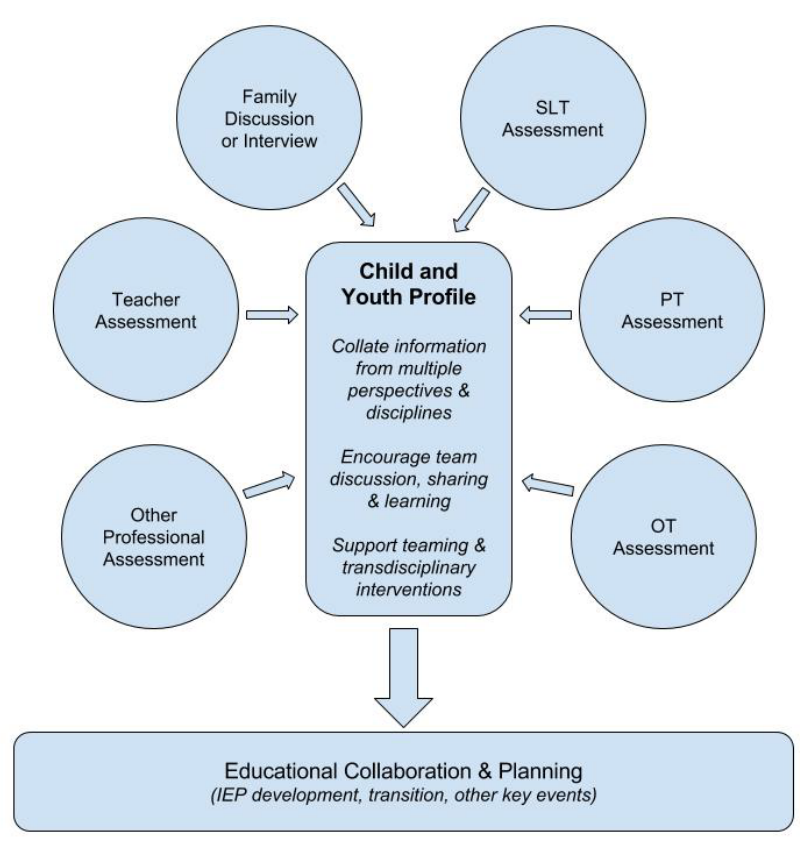

Figure 1. Framework for Child and Youth Profile.

Related to the collation of the information, the toolkit uses ratings across several of the forms and profiles. The purpose(s) of the ratings are to provide summary statements about children's functioning across areas and serve as a catalyst for conversation when team members have differing perspectives about children's abilities or limitations that are difficult to capture in a rating alone. Although challenging, ratings can also force conversations about team members' differing perspectives of children's abilities in different contexts. This was observed in the setting in which the team member completed the toolkit with a mother and father, and all parties were surprised to find out that mum and dad would have applied very different ratings. The team member reported this resulted in a very productive conversation about the child. As we make revisions to the toolkit, we will continue to consider when ratings might be used and how to structure ratings in ways that are useful and meaningful. 
Perhaps the most encouraging feedback on the toolkit was that all of the participants found that by working through the profiles, they found gaps in their knowledge about the child and were encouraged to consider some aspects or undertake further assessments in areas that they had not previously considered. This suggests that the toolkit can be effective for helping team members to gain a more multi-dimensional understanding of a child. Our larger aim, however, will be for multi-dimensional understanding to also lead to cross-disciplinary collaboration and teaming for educational planning.

\section{CONCLUSIONS AND RECOMMENDATIONS}

Based on the findings from the pilot study, we believe that the toolkit does have the potential to be used to facilitate cross-disciplinary educational planning if some revisions are made and extra guidelines or training are implemented. We have, therefore, revised the toolkit and developed guidelines for future use.

Revisions include:

- Background Information. Adding space to selfidentify cultural identity, home language, funding category, living situation and provider of support services.

- Sensory Modality Profile. Replacing the ratings of the use of different sensory modalities with questions. Adding space to discuss sensory integration issues.

- Communication Profile. Adding additional functions of communication.

- Participation and Access Profile. Including questions as to the levels of participation and more generic categories in the educational setting form. Adding more examples in the home and community profile and eliminating the rating scales in both profiles.

- Functional Ability Profile. Revising the focus of the ratings to identify strengths and limitations across areas within the child; so that teams can build on a child's functional strengths while supporting the child's development in areas of need.

- Adaptations and Specialised Equipment Profile. Including a column to indicate any adaptations or equipment that may have been trialled previously. Including some additional items such as visual timetables and devices for environmental control.

- Interests and Preferences Profile. Refining the directions and providing additional explanation of some items.
- Priority Planning Pages. Expanding the summary form and encouraging teams to use the educational planning form as well as their existing structures for individual education plans (IEPs) or other educational planning tools.

To support future use of the Child and Youth Profile, we have created a framework that shows how the profile might be used as a point of collation of information gathered from a range of perspectives and team members. As seen in Figure 1, relevant team members continue in-depth assessments in key areas appropriate for the child. This might include specialist assessments and interviews with parents. Summary information from these assessments and other information from those working with a child are then collated and discussed using the toolkit. The extent to which one person might be responsible for connecting with team members to provide input into the completion of forms or whether each team member completes section(s) relevant for them prior to a team meeting for discussion will depend on the dynamic and structure of the team. Making time to meet together, or with as many team members as possible, is critical for team sharing and discussion to help develop new, shared, and multi-dimensional understandings of the child (Simeonsson, 2009). Although it takes time, these discussions support the development and implementation of robust, integrated educational plans and interventions in line with Collaboration for Success (Ministry of Education, 2011) that are useful and effective for children and families.

In this article we have discussed our work with the Child and Youth Profile to date. This work is ongoing and we fully acknowledge that the toolkit has its limitations and requires trained teams to ensure appropriate use. In fact, all informationgathering and collation systems have limitations and potential for misuse (Florian et al., 2006). It is unrealistic, however, to abandon the use of these systems as practical tools. Awareness of the inherent limitations, clear articulation of the purposes, careful attention to the processes, and effective collaboration are needed to ensure tools like the Child and Youth Profile result in positive integrated educational outcomes (Florian et al., 2006). Crossdisciplinary collaboration can be challenging, but the benefits to children, families and education professionals can also be rewarding. For the benefit of children and youth with disabilities, we offer the New Zealand Child and Youth Profile as one tool that might help facilitate cross-disciplinary approaches to educational planning. 


\section{REFERENCES}

American Association on Intellectual and Developmental Disabilities (2010). Intellectual disability: Definition, classification, and systems of supports. Washington, DC: AAIDD.

Annan, J., \& Mentis M. (2013). Shifting perspectives to shape inclusive practices. In Centre of Excellence for Research in Inclusive Education (Ed.), Inclusive education: Perspectives on professional practice (pp. 25-39). Auckland, NZ: Dunmore.

Bjorck-Akesson, E., Wilder, J., Granlund, M., Pless, M., Simeonsson, R. J., Adolfsson, M., \& Lillvist, A. (2010). The international classification of functioning, disability and health and the version for children and youth as a tool in child habilitation/early childhood intervention: Feasibility and usefulness as a common language and frame of reference for practice. Disability and Rehabilitation, 32, 125-138. doi.10.3109/0963828 8.2010.516787

Budd, J. (2016). Using meta-perspectives to improve equity and inclusion. New Zealand Journal of Educational Studies, 51(2), 227-244. doi.10.1007/ s40481-016-0060-1

Burke, P. J., \& Ruedel, K. (2008). Disability classification, categorization in education: A US perspective. In L. Florian \& M. McLaughlin (Eds.), Disability classification in education: Issues and perspectives (pp. 68-76). Thousand Oaks, CA: Corwin Press.

Cameron, C. A. (2008). Identification and classification of childhood developmental difficulties in the context of attachment relationships. Journal of Canadian Child Adolescent Psychiatry, 17, 58-67.

Cramm, H., Aiken, A. B., \& Stewart, D. (2012). Perspectives on the international classification of functioning, disability, and health: Child and youth version (ICF-CY) and occupational therapy practice. Physical and Occupational Therapy in Pediatrics, Early Online, 1-16. doi.10.3109/01942 638.2012.680009

Conklin, J. (2005). Wicked problems and social complexity. In J. Conklin (Ed.), Dialogue mapping: Building shared understanding of wicked problems (pp. 3-40). Chichester, UK: Wiley.

Demchack, M., Alden, P., Bergin, C., Ting, S., \& Lacey, S. (1995). Evaluating transdiciplinary teaming for students with disabilities. Rural Special Education Quarterly, 14(1), 24-32.
Florian, L., Hollenweger, J., Simeonsson, R. J., Wedell, K., Riddell, S., Terzi, L., \& Holland, A. (2006). Cross-cultural perspectives on the classification of children with disabilities. The Journal of Special Education, 40, 36-45. doi.10.11 77/00224669060400010401

Hinrichs, C. (2008). Interdisciplinarity and boundary work: Challenges and opportunities for agrifood studies. Agricultural and Human Values, 25, 209213. doi:10.1007/s10460-008-9118-0

Lollar, D. J., \& Simeonsson, R. J. (2005). Diagnosis to function: Classification for children and youths. Developmental and Behavioral Pediatrics, 26, 323330. doi.10.1097/00004703-200508000-00012

McLaughlin, T., Snyder, P., \& Algina, J. (2015). Using functional profiles to characterize childhood disabilities. Exceptional Children, 81, 471-488. doi.10.1177/0014402914563696

Ministry of Education (2011). Collaboration for success: Individual education plans. Wellington, NZ: Ministry of Education.

Peterson, D. B. (2005). International classification of functioning, disability and health: An introduction for rehabilitation psychologists. Rehabilitation Psychology, 50, 105-112. doi.10.1037/00905550.50.2.105

Rosenbaum, P., \& Stewart, D. (2004). The world health organization international classification of functioning, disability and health: A model to guide clinical thinking, practice, and research in the field of cerebral palsy. Seminars in Pediatric Neurology, 11, 5-10. doi.10.1016/j.spen.2004.01.002

Simeonsson, R. J. (2009). ICF-CY: A universal tool for documentation of disability. Journal of Policy and Practice in Intellectual Disability, 6, 70-72. doi.10.1111/j.1741-1130-2009.00215x

Simeonsson, R. J., Bailey, D. B., Smith, T. M., \& Buysse, V. (1995). Young children with disabilities: Functional assessment by teachers. Journal of Development and Physical Disabilities, 7, 267284. doi.1007/BF02578431

Simeonsson, R. J., Leonardi, M., Lollar, D., BjorckAkesson, E., Hollenweger, J., \& Martinuzzi, A. (2003). Applying the international classification of functioning, disability, and health (ICF) to measure childhood disability. Disability and Rehabilitation, 25, 602-610. doi.10.1080/0963828031000137117 
Terzi. L. (2008). Beyond the dilemma of difference: The capability approach in disability and special needs education. In L. Florian \& M. McLaughlin (Eds.), Disability classification in education: Issues and perspectives (pp. 244-262). Thousand Oaks, CA: Corwin Press.

World Health Organization (2007). International classification of functioning, disability and health: Children and youth version. Geneva, Switzerland: WHO Press.

\section{AUTHOR PROFILES}

Tara Way McLaughlin

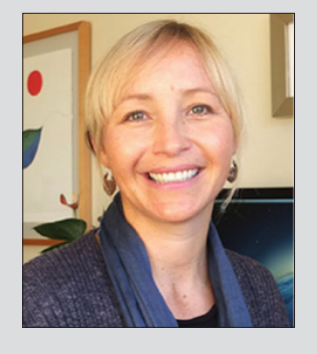

Tara Way McLaughlin is a senior lecturer in Early Years at Massey University. Her current research interests relate to (a) teaching practices that support children's learning and social-emotional competence within inclusive environments; (b) professional learning opportunities for early childhood teachers and teams; and (c) assessment practices in early childhood and early intervention.

Email: T.W.McLaughlin@massey.ac.nz

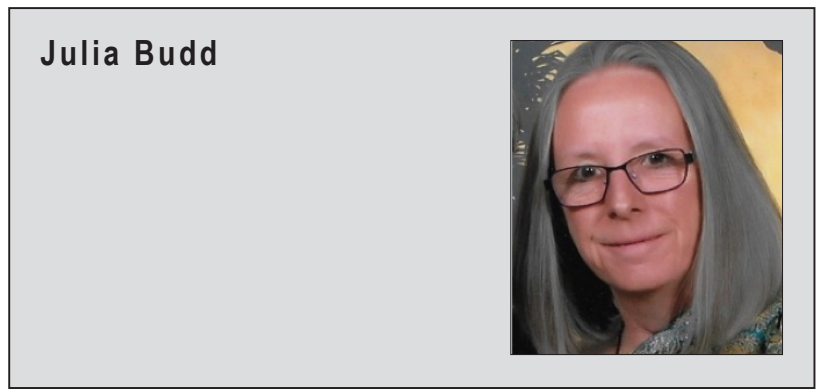

Julia Budd is a lecturer on the Specialist Teaching programme at Massey University. Her main areas of interest are in education of blind and low vision learners. Her doctoral study was in the field of interprofessional collaboration and she is passionate about helping a range of professionals collaborate and integrate their knowledge and expertise to improve outcomes for learners.

Email: J.M.Budd@massey.ac.nz

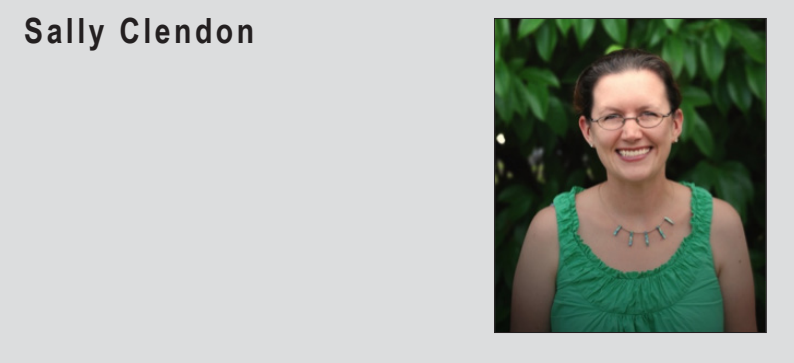

Sally Clendon is a senior lecturer in Speech and Language Therapy at Massey University. Her research and clinical interests are focused around supporting communication and literacy learning for children with complex communication needs who use augmentative and alternative communication.

Email: S.Clendon@massey.ac.nz 